\title{
Adverse events in contemporary continuous-flow left ventricular assist devices: A multi-institutional comparison shows significant differences
}

John M. Stulak, MD, ${ }^{\mathrm{a}}$ Mary E. Davis, MS, ${ }^{\mathrm{b}}$ Nicholas Haglund, MD, ${ }^{\mathrm{b}}$ Shannon Dunlay, MD, Jennifer Cowger, MD, ${ }^{\mathrm{c}}$ Palak Shah, MD, ${ }^{\mathrm{d}}$ Francis D. Pagani, MD, PhD, ${ }^{\mathrm{e}}$ Keith D. Aaronson, MD, ${ }^{\mathrm{e}}$ and Simon Maltais, $\mathrm{MD}, \mathrm{PhD}^{\mathrm{a}}$

\section{ABSTRACT}

Objectives: We review differences in the incidence and timing of adverse events in patients implanted with continuous-flow left ventricular assist devices (LVADs), using the Mechanical Circulatory Support Research Network registry.

Methods: From May 2004 to September 2014, a total of 734 patients (591 men; median age: 59 years) underwent primary continuous-flow LVAD implantation at our institutions. Patients implanted with the HeartMate II (HMII) (560 [76\%] patients), compared with the HeartWare ventricular assist device (HVAD; 174 $[24 \%])$ were more often receiving destination therapy $(47 \%$ vs $20 \% ; P<.01)$, had a lower preoperative creatinine level (1.2 vs $1.3 ; P=.01)$, and had less median preoperative right ventricular dysfunction (mild vs moderate; $P<.01$ ). Ischemic etiology, prior sternotomy, and median INTERMACS profile were similar.

Results: Overall mortality was 54 of $734(7.4 \%)$; 41 of 560 (7.3\%) in the HMII group, and 13 of $174(7.5 \%)$ in the HVAD group $(P=.95)$. Follow-up was available in $100 \%$ of early survivors for a median of 1 year (max: 10 years) and a total of 1120 patient-years of support (HMII: 940 patient-years [median: 1.1 years, max: 5.3 years] and HVAD: 180 patient-years [median: 0.6 year, max: 10.4 years]). On multivariable analysis, GI bleeding $(P=.63)$, any infection $(P=.32)$, driveline infection $(P=.10)$, and pump thrombus $(P=.64)$ were similar between devices while HeartWare HVAD was associated with higher risk of stroke (HR: 1.8, [1.25, 2.5], $P=.003$ ).

Conclusions: In this pooled analysis, a trend was found for higher incidence of percutaneous driveline infections in patients treated with the HMII; a higher incidence of stroke and time-related cumulative risk of any infection and stroke was found in patients treated with the HVAD, which was independently associated with higher stroke risk. (J Thorac Cardiovasc Surg 2016;151:177-89)

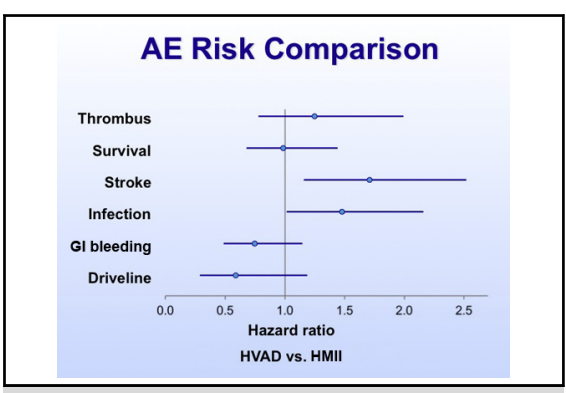

Adverse event risk for the HVAD versus the HMII LVADs.

\section{Central Message}

Patients treated with the HVAD, versus HMII had a higher incidence of stroke and timerelated cumulative risk of any infection and stroke.

\section{Perspective}

Very few large, multicenter comparisons have been done between contemporary axial- and centrifugal-flow left ventricular assist devices. Understanding these differences can substantially enhance preoperative counseling, as well as postoperative monitoring, of these patients.

See Editorial Commentary page 190.

See Editorials pages 10 and 13.

\footnotetext{
From the ${ }^{\mathrm{a} D i v i s i o n}$ of Cardiovascular Surgery, Mayo Clinic College of Medicine, Rochester, Minn; ${ }^{\mathrm{b}}$ Department of Cardiac Surgery, Vanderbilt Heart and Vascular Institute, Nashville, Tenn; ${ }^{c}$ Division of Cardiovascular Diseases, St Vincent Heart Center, Indianapolis, Ind; ${ }^{\mathrm{d} D i v i s i o n}$ of Cardiovascular Diseases, Inova Fairfax Hospital, Fairfax, Va; and ${ }^{\mathrm{e}}$ Department of Cardiac Surgery, University of Michigan Health System, Ann Arbor, Mich.

Read at the 95th Annual Meeting of The American Association for Thoracic Surgery, Seattle, Washington, April 25-29, 2015.

Received for publication May 9, 2015; revisions received Aug 27, 2015; accepted for publication Sept 6, 2015; available ahead of print Nov 4, 2015

Address for reprints: John M. Stulak, MD, Division of Cardiovascular Surgery, Mayo Clinic College of Medicine, 200 First St SW, Rochester, MN 55905 (E-mail: stulak. john@mayo.edu).

$0022-5223 / \$ 36.00$

Copyright (c) 2016 by The American Association for Thoracic Surgery

http://dx.doi.org/10.1016/j.jtcvs.2015.09.100
}

The largest analysis to date for patients undergoing implantation of a left ventricular assist device (LVAD) approved by the Food and Drug Administration was summarized in the sixth annual report of the Interagency Registry for Mechanically Assisted Circulatory Support (INTERMACS); it presents an 8-year enrollment of $>10,000$ patients. ${ }^{1}$ The accrual rate noted in this report now exceeds 2000 patients per year. Despite the large amounts of pooled data for these patients, from both the INTERMACS registry and multicenter clinical trials and postapproval analyses, very few direct comparisons of second- and third-generation LVADs have been done. The reports include a comparison of the 


$\begin{array}{ll}\text { Abbreviations and } & \text { Acronyms } \\ \mathrm{CI} & =\text { confidence interval } \\ \mathrm{GI} & =\text { gastrointestinal } \\ \mathrm{HMII} & =\text { HeartMate II (LVAD) } \\ \mathrm{HR} & =\text { hazard ratio } \\ \mathrm{HVAD} & \text { HeartWare Ventricular Assist } \\ & \text { Device (LVAD) } \\ \mathrm{INR} & =\text { international normalized ratio } \\ \mathrm{INTERMACS}= & \text { Interagency Registry for } \\ & \text { Mechanically Assisted Circulatory } \\ & \text { Support } \\ \text { LVAD } & \text { left ventricular assist device } \\ & \end{array}$

third-generation HeartWare Ventricular Assist System (HVAD; HeartWare Inc, Framingham, Mass) against a control group of commercially available devices from the INTERMACS registry, ${ }^{2}$ a single-institution experience comparing patient outcomes for the HeartMate II (HMII; Thoratec Corporation, Pleasanton, Calif) and the HVAD, ${ }^{3}$ and the preliminary ${ }^{4}$ and full set $^{5}$ of data from the Endurance randomized, controlled clinical trial. Aside from these reports, very few data are available from specific comparisons of these devices.

Because very few reports are available from large, multicenter, collaborative efforts analyzing contemporary outcomes in a real-world setting, we sought to evaluate outcomes in our large cohort with an "all-comers" strategy for patients implanted with a continuous-flow LVAD. We report differences in preoperative clinical characteristics, operative procedures, and early and late outcomes, including overall survival, gastrointestinal (GI) bleeding, infection, percutaneous driveline infection, stroke, and pump thrombus. We performed a multivariable analysis, to identify independent predictors of each late outcome, and specifically device type, to detect potential independent associations with late outcomes.

\section{METHODS \\ Patients}

The data collection and analysis were performed after informed patient consent was obtained and the study had been approved by the University of Michigan, Mayo Clinic College of Medicine, and Vanderbilt Heart and Vascular Institute Institutional Review Board. Between May 2004 and September 2014, a total of 734 patients underwent primary continuous-flow LVAD at the centers that comprise the Mechanical Circulatory Support Research Network (University of Michigan Health System, Mayo Clinic College of Medicine, and Vanderbilt Heart and Vascular Institute). The median age at the time of operation for the entire cohort was 59 years (range: $18-82$ years); 591 (80\%) were men. Devices implanted include the HMII in $560(76 \%)$, and the HVAD in $174(24 \%)$. Stratification by device type revealed several significant differences in preoperative clinical characteristics (Table 1).

In general, for all institutions, lactate dehydrogenase is monitored weekly for the first month, monthly thereafter to the 6-month point, and then every 6 months. International normalized ratio (INR) values are closely monitored by institutional anticoagulation programs, and patients are routinely bridged with unfractionated intravenous heparin if INR
$<2.0$. All patients with the HVAD were maintained on $325 \mathrm{mg}$ of aspirin. Dipyridamole is used almost routinely by the University of Michigan before therapeutic INR, and selectively by the other institutions. Plavix was utilized on an individual basis if pump thrombus or hemolysis was observed; it is not used routinely, especially in bridge-to-transplant patients, as this would pose a greater bleeding risk at transplant. We have introduced use of bridging with low-molecular weight heparin in stable outpatients if the INR falls to $<2.0$ and remains $>1.7$, to avoid readmission.

All adverse events were defined according to the standard INTERMACS definition used during the time period of implantation. This cohort represents patients receiving continuous-flow LVAD implantation during various eras that span several important clinical trials, including the HeartMate II DT (destination therapy) trial, the HeartWare BTT (bridge to transplant) trial (ADVANCE), and the HeartWare DT (destination therapy) trial (ENDURANCE I). A segment of this population was implanted in a commercial setting, after HMII destination therapy approval in 2009, and HeartWare bridge to transplant approval in 2012.

\section{Statistical Analysis}

Demographic and other patient-related data were obtained from the University of Michigan, Mayo Clinic College of Medicine, and the Vanderbilt Heart and Vascular Institute medical record, along with our prospectively collected clinical databases. Follow-up information was obtained from subsequent clinic visits and written correspondence from local physicians. Data were expressed as either mean $\pm \mathrm{SE}$ of the mean, for normally distributed data, or median with range for non-normally distributed data. Normality of continuous variables was assessed using the Shapiro-Wilk test. Mann-Whitney U and Wilcoxon-rank-sum were both used to compare significantly skewed continuous data.

Data between 2 groups were compared using $\chi^{2}$ analysis for continuous and dichotomous variables, respectively. A backward, stepwise Cox regression analysis was used to identify perioperative variables that independently affected outcomes. Variables significant in the univariate analysis were utilized during stepwise selection to create the final multivariable model.

Variables included in the univariate analysis included the following: device type (HMII, HVAD); age at implant; gender; implanting institution; device indication (bridge, destination); heart failure etiology (dilated, ischemic); preoperative atrial fibrillation; diabetes; hypertension; total bilirubin; creatinine; hemoglobin; INTERMACS profile; preoperative inotropes; preoperative intra-aortic balloon pump; preoperative tricuspid regurgitation grade; preoperative right ventricular dysfunction; redo sternotomy; tricuspid valve surgery; aortic valve surgery; and cardiopulmonary bypass time. In addition, for pump thrombus and stroke, the eras of preAugust 2011 and post-August 2011 were entered as variables for analysis.

Kaplan-Meier survival analysis was used to evaluate time-related outcomes and create plots, which were subsequently compared using the log-rank test. Early operative mortality was defined as death within 30 days of operation, or at any time during the index hospitalization. We employed pairwise deletion to impute missing data.

\section{RESULTS \\ Operative Data}

All patients underwent primary continuous-flow LVAD implantation at our respective institutions. Preoperative clinical characteristics differed when stratified by device type (Table 2); significantly more patients underwent tricuspid valve surgery in the HMII cohort $(n=207$; $38 \%)$ compared with the HVAD group $(n=34 ; 21 \%)$.

\section{Early Outcomes}

Overall mortality was 54 of $734(7.4 \%) ; 41$ of 560 $(7.3 \%)$ in the HMII group; and 13 of $174(7.5 \%)$ in the 
TABLE 1. Preoperative clinical characteristics of the study cohort

\begin{tabular}{|c|c|c|c|c|}
\hline Preoperative clinical characteristic & $\begin{array}{c}\text { HMII } \\
(\mathrm{n}=\mathbf{5 6 0})\end{array}$ & $\begin{array}{c}\text { HVAD } \\
(n=174)\end{array}$ & $\begin{array}{c}\text { Total } \\
(\mathbf{n}=734)\end{array}$ & $P$ value \\
\hline Age at implant (y), median (range) & $59.5(18.0-82.0)$ & $58.3(18.3-76.5)$ & $59.1(18.0-82.0)$ & .02 \\
\hline BMI $\left(\mathrm{kg} / \mathrm{m}^{2}\right)$, median (range) & $28.5(2.0-142.2)$ & $27.6(16.3-43.7)$ & $28.3(2.0-142.2)$ & .0321 \\
\hline INTERMACS profile & & & & .0453 \\
\hline Mean (SD) & $2.8(1.1)$ & $2.9(1.1)$ & $2.8(1.1)$ & \\
\hline 1 & $74(13)$ & $19(11)$ & - & .54 \\
\hline 2 & $125(22)$ & $25(14)$ & - & .03 \\
\hline 3 & $185(33)$ & $78(45)$ & - & .07 \\
\hline $4-7$ & $176(32)$ & $52(30)$ & - & .35 \\
\hline Total bilirubin, $(\mu \mathrm{mol} / \mathrm{L})$, mean $(\mathrm{SD})$ & $1.3(0.9)$ & $1.2(1.0)$ & $1.3(1.0)$ & .2481 \\
\hline Creatinine (mg/dL), mean (SD) & $1.4(0.5)$ & $1.3(0.5)$ & $1.4(0.5)$ & .0035 \\
\hline Gender & & & & .002 \\
\hline Male & $465(83)$ & $126(72)$ & $591(80)$ & \\
\hline Female & $95(17)$ & $48(28)$ & $143(20)$ & \\
\hline Heart failure etiology & & & & .52 \\
\hline Ischemic & $278(50)$ & $82(47)$ & $360(50)$ & \\
\hline Non-ischemic & $276(50)$ & $91(53)$ & $367(50)$ & \\
\hline Hypertension & $263(47)$ & $101(58)$ & $364(50)$ & .013 \\
\hline Atrial fibrillation & $197(35)$ & $72(41)$ & $269(37)$ & .14 \\
\hline Diabetes & $194(35)$ & $57(36)$ & $251(35)$ & .75 \\
\hline Device indication & & & & $<.001$ \\
\hline Bridge-to-transplant & $296(53)$ & $140(81)$ & $436(60)$ & \\
\hline Destination therapy & $264(47)$ & $33(19)$ & $297(40)$ & \\
\hline Preoperative inotropes & $425(76.4)$ & $126(74.6)$ & $551(76.0)$ & .62 \\
\hline RVSWI (g-m/ $/ \mathrm{m}^{2} /$ beat), median (range) & $514(103-1009)$ & $464(100-1200)$ & $492(100-1200)$ & .18 \\
\hline PA pressure ( $\mathrm{mm} \mathrm{Hg}$ ), mean (range) & $34(10-70)$ & $31(12-57)$ & $33(10-70)$ & .004 \\
\hline PCWP (mm Hg), median (range) & $22(5-52)$ & $20(3-43)$ & $22(3-52)$ & .03 \\
\hline Device IABP & $263(47.8)$ & $64(36.8)$ & $327(45.2)$ & .011 \\
\hline
\end{tabular}

HVAD group $(P=.95)$. In the entire cohort, total median length of stay was 18 days (range: 0-384 days); postoperative inotrope use was 5 days (range: 0-57 days); and need for dialysis was 75 of $734(10 \%)$. With stratification by device type, early need for dialysis $(10 \%$ vs $10 \% ; P=.96)$ and length of postoperative inotrope therapy $(6[0-31]$ days vs 5 [0-57] days; $P=.3$ ) were similar between the HMII and HVAD groups, respectively; total median length of stay was significantly longer in the HMII group (20 [0384] days vs 16 [0-164] days; $P<.001)$.

TABLE 2. Operative clinical characteristics in the study cohort

\begin{tabular}{lcccc}
\hline \multicolumn{1}{c}{ Treatment } & $\begin{array}{c}\text { HMII } \\
(\mathbf{n}=\mathbf{5 6 0})\end{array}$ & $\begin{array}{c}\text { HVAD } \\
(\mathbf{n}=\mathbf{1 7 4})\end{array}$ & $\begin{array}{c}\text { Total } \\
(\mathbf{n}=\mathbf{7 3 4})\end{array}$ & $\boldsymbol{P}$ value \\
\hline Redo sternotomy & $185(33)$ & $53(31)$ & $238(33)$ & .51 \\
TV surgery & $207(38)$ & $34(21)$ & $241(34)$ & $<.001$ \\
AV surgery & $44(9)$ & $6(5)$ & $50(8)$ & .22 \\
Temporary RVAD & $27(5)$ & $6(4)$ & $33(5)$ & .44 \\
\hline
\end{tabular}

Values are $\mathrm{n}(\%)$, unless otherwise indicated. HMII, HeartMate II; HVAD, HeartWare ventricular assist device; $T V$, tricuspid valve; $A V$, aortic valve; $R V A D$, right-ventricular assist device.

\section{Late Outcomes and Overall Survival}

Follow-up was available in all 680 early survivors, for a median duration of 1 year (maximum: 10.4 years). Overall survival for the entire cohort was $83 \%, 62 \%$, and $45 \%$, at 1 , 3 , and 5 years, respectively. No difference was found in overall survival, with data stratified by device type $(P=.96)$ (Figure 1). Independent predictors of worse late survival that were identified in a multivariable model included cardiopulmonary bypass time (hazard ratio [HR], with 95\% confidence interval [CI]: $1.1[1.05,1.15]$; $P<.001)$ and preoperative creatinine (HR: $1.6[1.28$, 2.02]; $P<.001)$. When device type was forced into the multivariable model, neither were independently associated with worse late survival $(P=.27)$.

\section{Gastrointestinal Bleeding}

Cumulative risk of gastrointestional (GI) bleeding for the entire cohort was $21 \%, 27 \%$ and $31 \%$, at 1,3 , and 5 years, respectively. No significant difference in time-related cumulative risk of GI bleeding was found, with data stratified by device type $(P=.18)$ (Figure 2$)$. Independent predictors of GI bleeding identified in the multivariable model 


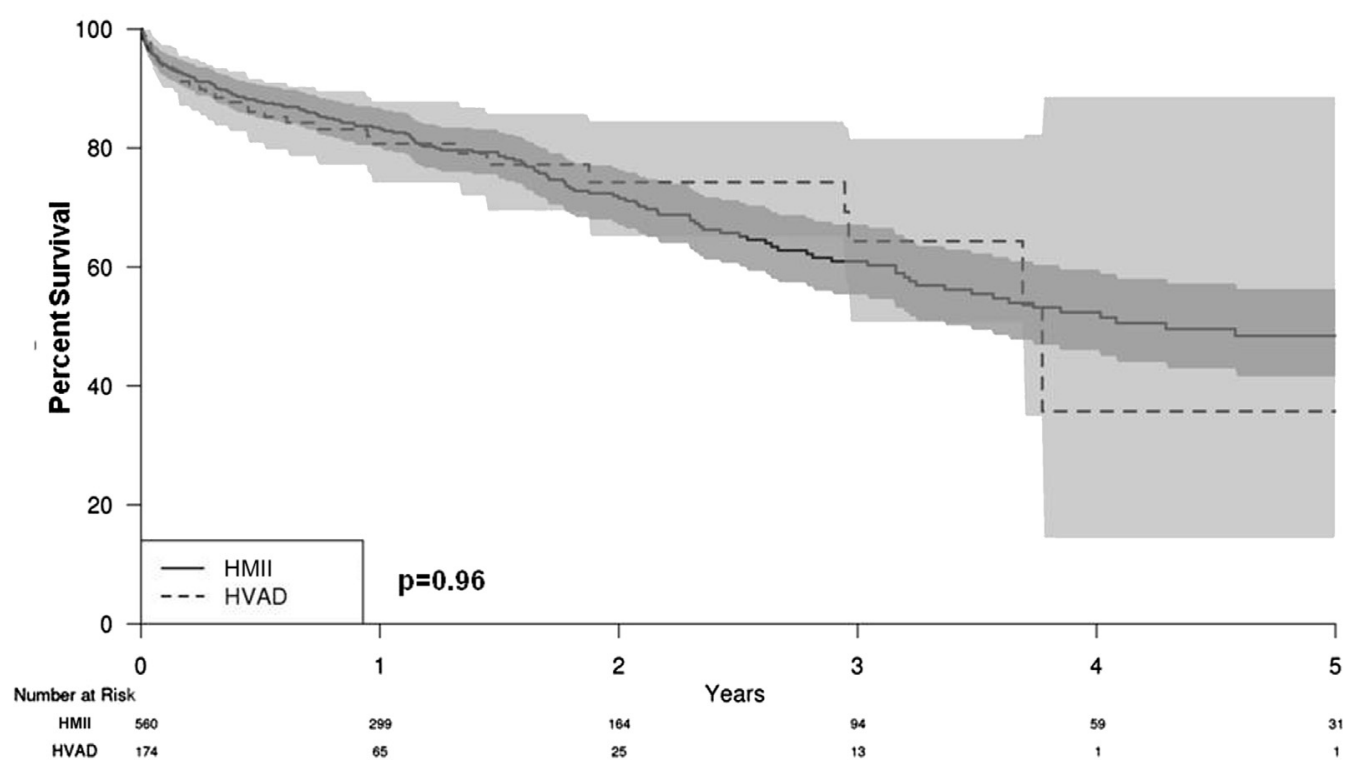

FIGURE 1. Overall survival for patients undergoing HMII (solid line) versus HVAD (dotted line) implantation; no difference was found, with data stratified by device type $(P=.96)$. HMII, HeartMate II; HVAD, HeartWare ventricular assist device.

included older age at implant (hazard ratio [95\% CI]: 1.05 $[1.03,1.06] ; P<.001)$; preoperative inotrope use (HR [95\% CI]: $1.7[1.2,2.4] ; P=.003$ ); redo sternotomy (HR [95\% CI]: $0.6[0.44,0.86] ; P<.001)$; and preoperative creatinine (HR [95\% CI]: $1.3[1.02,1.7] ; P=.03$ ). When device type was forced into the multivariable model, neither was independently associated with GI bleeding $(P=.63)$.

\section{Any Infection}

Cumulative risk of any infection for the entire cohort was $17 \%, 33 \%$, and $45 \%$, at 1,3 , and 5 years, respectively. The types of non-device-related infections for the entire cohort included bacteremia/sepsis in 24 patients, urinary tract in 14 , pulmonary in 13 , sternal wound without mediastinitis in 10 , GI in 6, peripherally inserted central catheter line in 3, implantable cardioverter defibrillator lead in 3, fever of unknown origin in 3 , cellulitis in 2 , and nonsternal osteomyelitis in 2. The time-related cumulative risk of any infection was significantly higher with the HVAD when stratified by device type $(P=.039)$ (Figure 3$)$. Independent predictors of any infection identified on a multivariable model included: older age at implant (HR [95\% CI]: 0.97

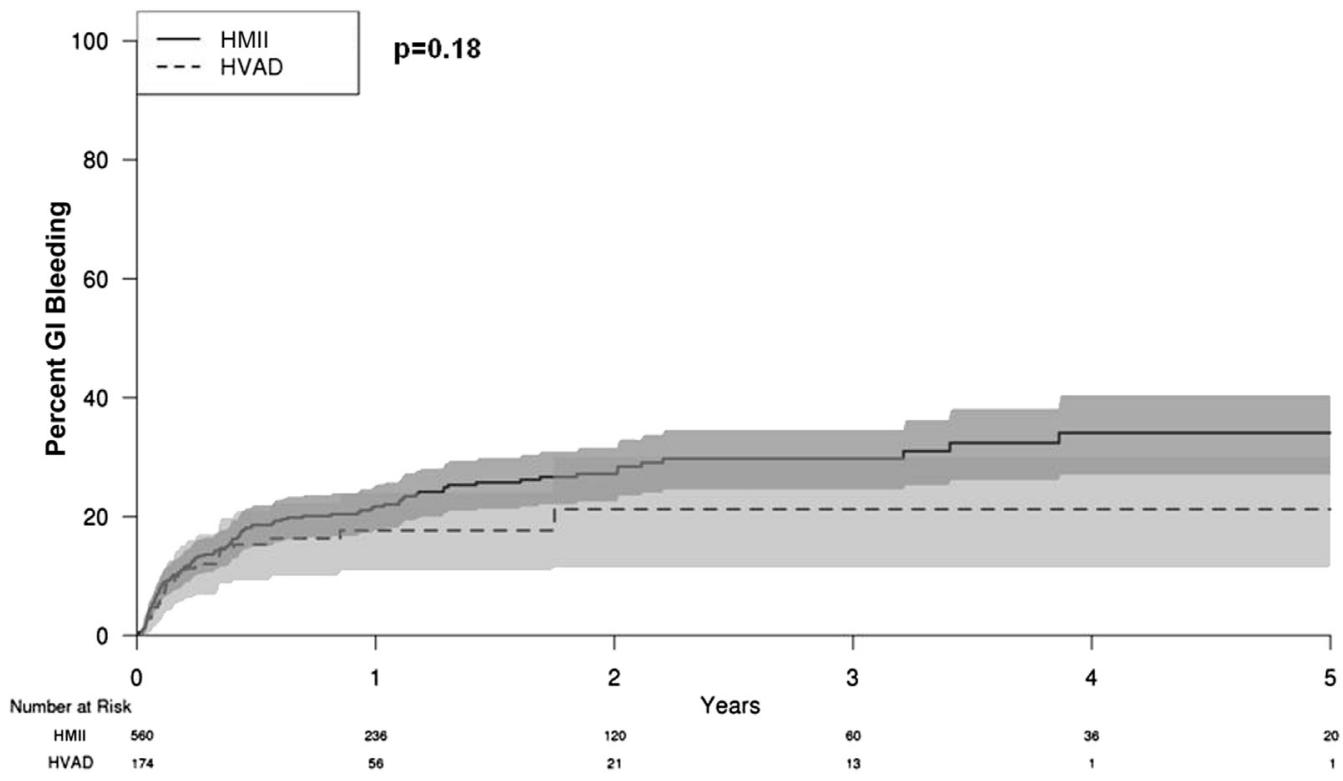

FIGURE 2. Time-related cumulative risk of GI bleeding for patients undergoing HMII (solid line) versus HVAD (dotted line) implantation; no significant difference was found, with data stratified by device type $(P=.18)$. HMII, HeartMate II; HVAD, HeartWare ventricular assist device; GI, gastrointestinal. 


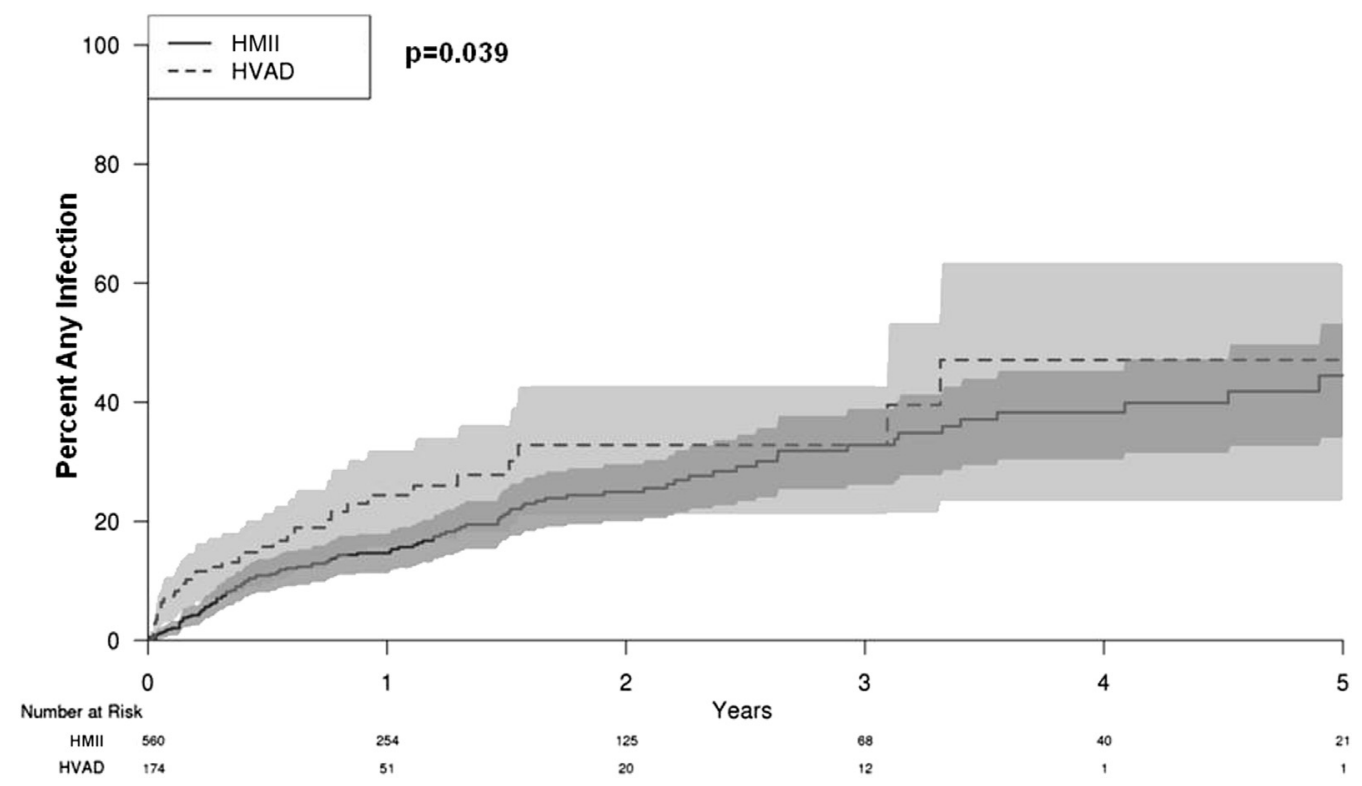

FIGURE 3. Time-related cumulative risk of any infection for patients undergoing HMII (solid line) versus HVAD (dotted line) implantation; the risk was significantly higher with the HVAD, with stratification by device type $(P=.039)$. HMII, HeartMate II; HVAD, HeartWare ventricular assist device.

[0.96, 0.99]; $P<.001$ ); hypertension (HR [95\% CI]: 0.66 $[0.46,0.93] ; P=.018$ ); and tricuspid valve surgery (HR [95\% CI]: $1.9[1.3,2.9] ; P=.0023)$. Neither device type was independently associated with any infection $(P=.32)$.

\section{Percutaneous Driveline Infection}

Cumulative risk of percutaneous driveline infection for the entire cohort was $7 \%, 20 \%$, and $29 \%$, at 1,3 , and 5 years, respectively. No difference was found in timerelated cumulative risk of percutaneous driveline infection, with data stratified by device type $(P=.14)$ (Figure 4).
Older age at implant (HR [95\% CI]: 0.98 [0.96, 0.99]; $P=.001)$ was the only independent predictor of percutaneous driveline infection identified in the multivariable model. When device type was forced into the multivariable model, neither was independently associated with percutaneous driveline infection $(P=.10)$.

\section{Stroke}

Cumulative risk of stroke for the entire cohort was $15 \%$, $33 \%$, and $39 \%$ at 1,3 , and 5 years, respectively. The timerelated cumulative risk of stroke was significantly higher

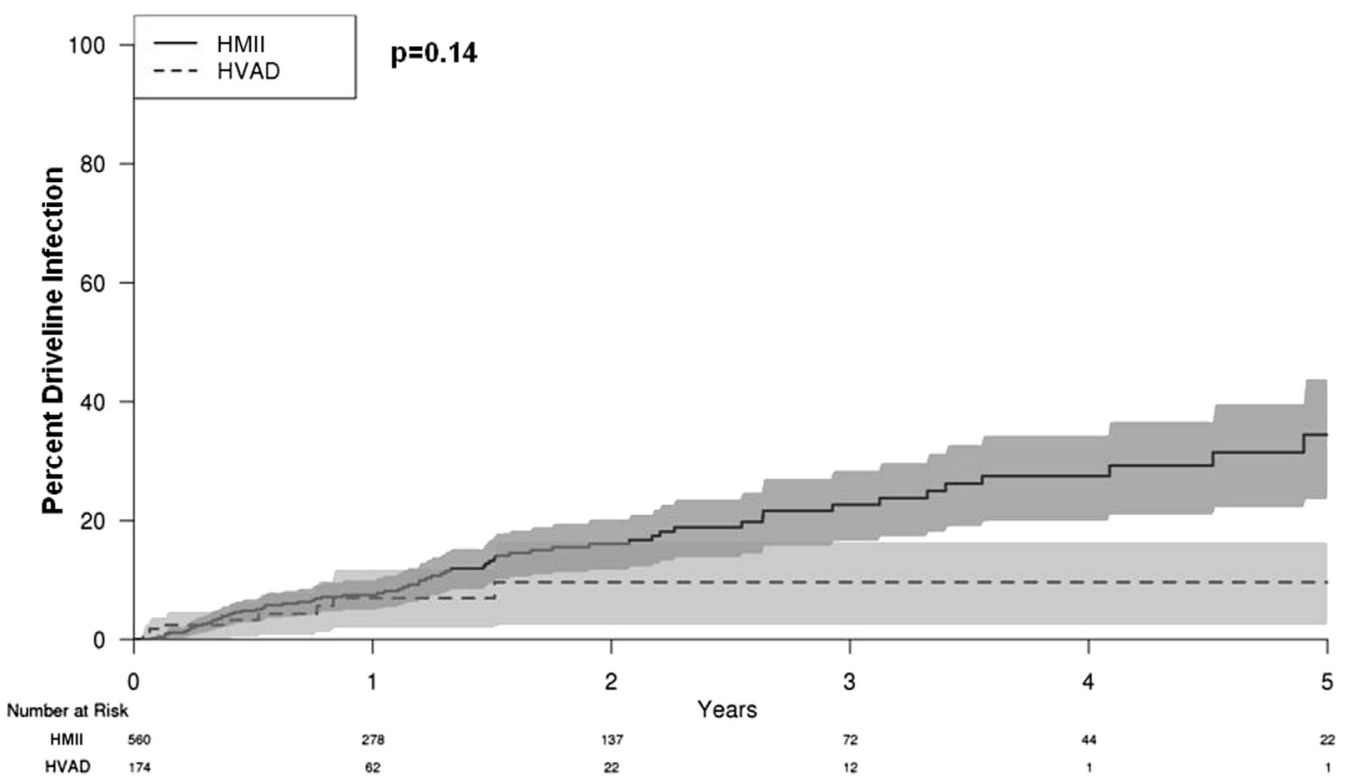

FIGURE 4. Time-related cumulative risk of percutaneous driveline infection for patients undergoing HMII (solid line) versus HVAD (dotted line) implantation; no difference was found, with data stratified by device type $(P=.14)$. HMII, HeartMate II; HVAD, HeartWare ventricular assist device. 

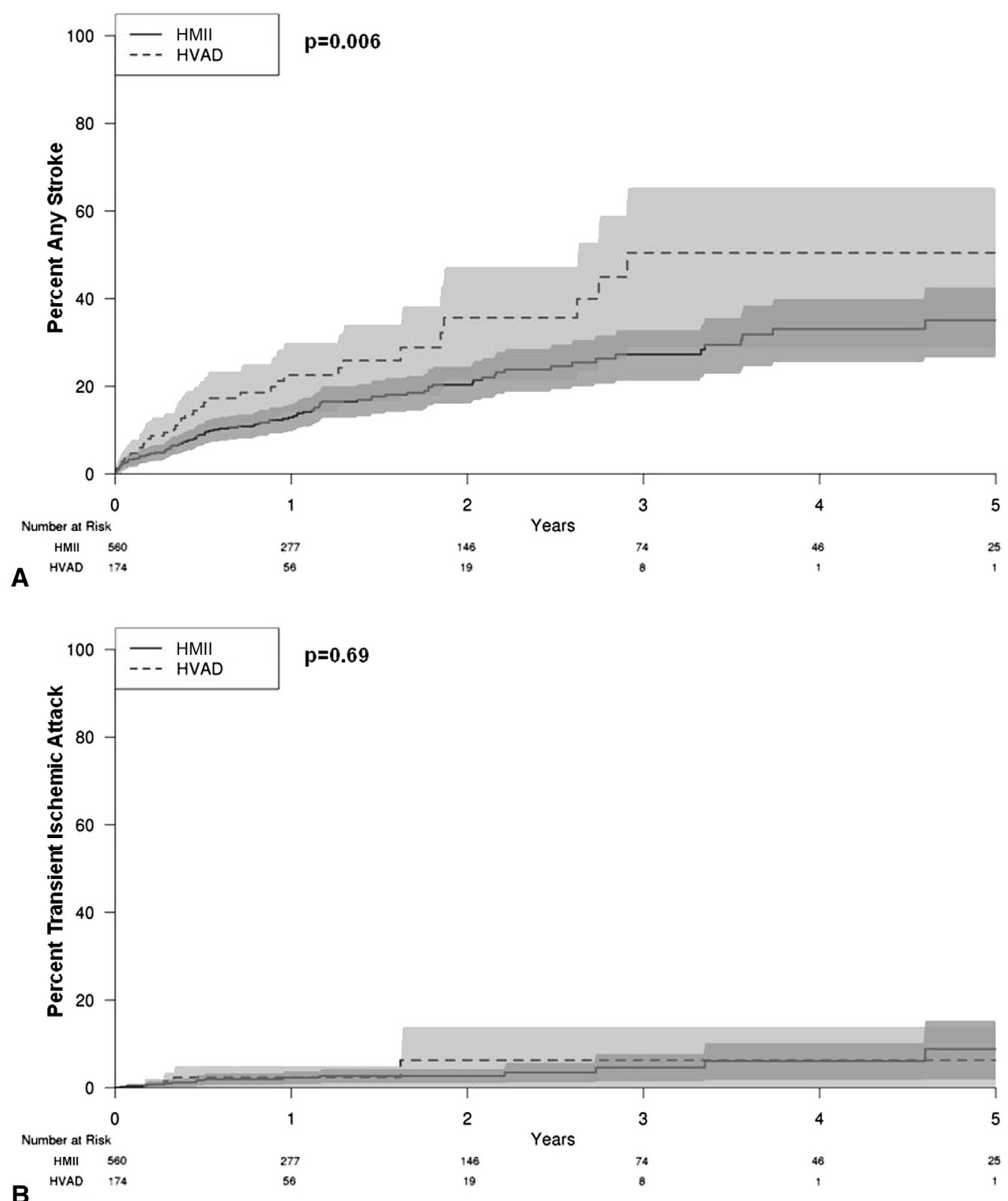

FIGURE 5. A, Time-related cumulative risk of stroke for patients undergoing HMII (solid line) versus HVAD (dotted line) implantation; a significantly higher risk of stroke was found with the HVAD, with data stratified by device type $(P=.006)$. B, No significant difference between devices was found in the cumulative risk of transient ischemic attack $(P=.69)$. C, No significant difference was found between devices in the cumulative risk of ischemic stroke $(P=.17)$. D, The cumulative risk of hemorrhagic stroke was significantly higher with the HVAD $(P=.006)$. HMII, HeartMate II; HVAD, HeartWare ventricular assist device.

with the HVAD, when stratified by device type $(P=.006)$ (Figure $5, A$ ). No difference was found in cumulative risk of transient ischemic attack (Figure 5, B) or ischemic stroke (Figure 5,C) between devices, whereas cumulative risk of hemorrhagic stroke was significantly higher with the HVAD (Figure 5, D).

Because of a major manufacturing change in the sintering of the HVAD, put into effect beginning in August 2011, we have performed further analysis of the cumulative incidence of stroke for patients who were implanted with the HVAD before and after this change. The 2-year cumulative risk of stroke after HVAD implantation was $36 \%$ in patients implanted before, versus $28 \%$ for those implanted after, August 2011. This difference was not statistically significant $(P=.33)$. Ischemic heart failure etiology (HR [95\% $\mathrm{CI}]: 1.6[1.1,2.3] ; P=.007)$ and HVAD were associated with a higher cumulative risk of stroke (HR $[95 \% \mathrm{CI}]$ : $1.8[1.25,2.5] ; P=.003)$. 

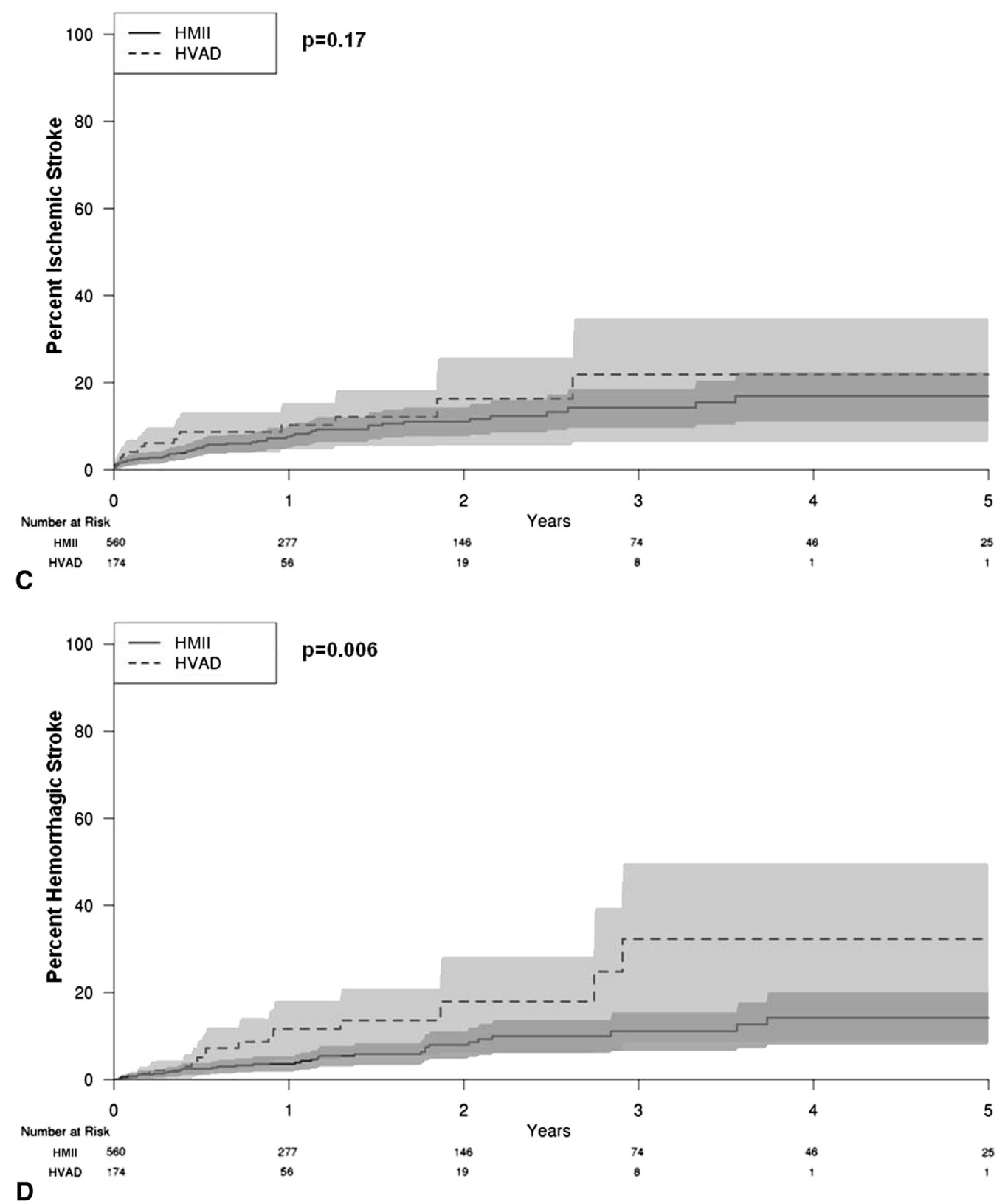

FIGURE 5. (Continued).

\section{Pump Thrombus}

Cumulative risk of pump thrombus for the entire cohort was $14 \%, 24 \%$, and $25 \%$, at 1,3 , and 5 years, respectively. No difference was found in the time-related cumulative risk of pump thrombus, with stratification by device type $(P=.35)$ (Figure 6, $A)$; no difference was found in cumulative risk of pump thrombus according to era of implantation (before and after August 2011; $P=.33$ ) (Figure 6, B). For HVAD patients, the 2-year cumulative risk of pump thrombus was $26 \%$ for implantation before sintering, and $22 \%$ after sintering $(P=.36)$; furthermore, era of implantation was not a significant predictor of pump thrombus in HVAD patients.

However, in examining the true incidence rate, pump thrombosis occurred in 10 of 28 patients (36\%), and in 11 of $146(7.5 \%)$ patients implanted before versus after August 2011, respectively. A lower INTERMACS profile (HR $[95 \% \mathrm{CI}]: 1.2[1.02,1.4] ; P=.03)$ was the only independent predictor of pump thrombus identified in the multivariable model. When device type was forced into the multivariable model, neither was independently associated with pump thrombus $(P=.64)$. 

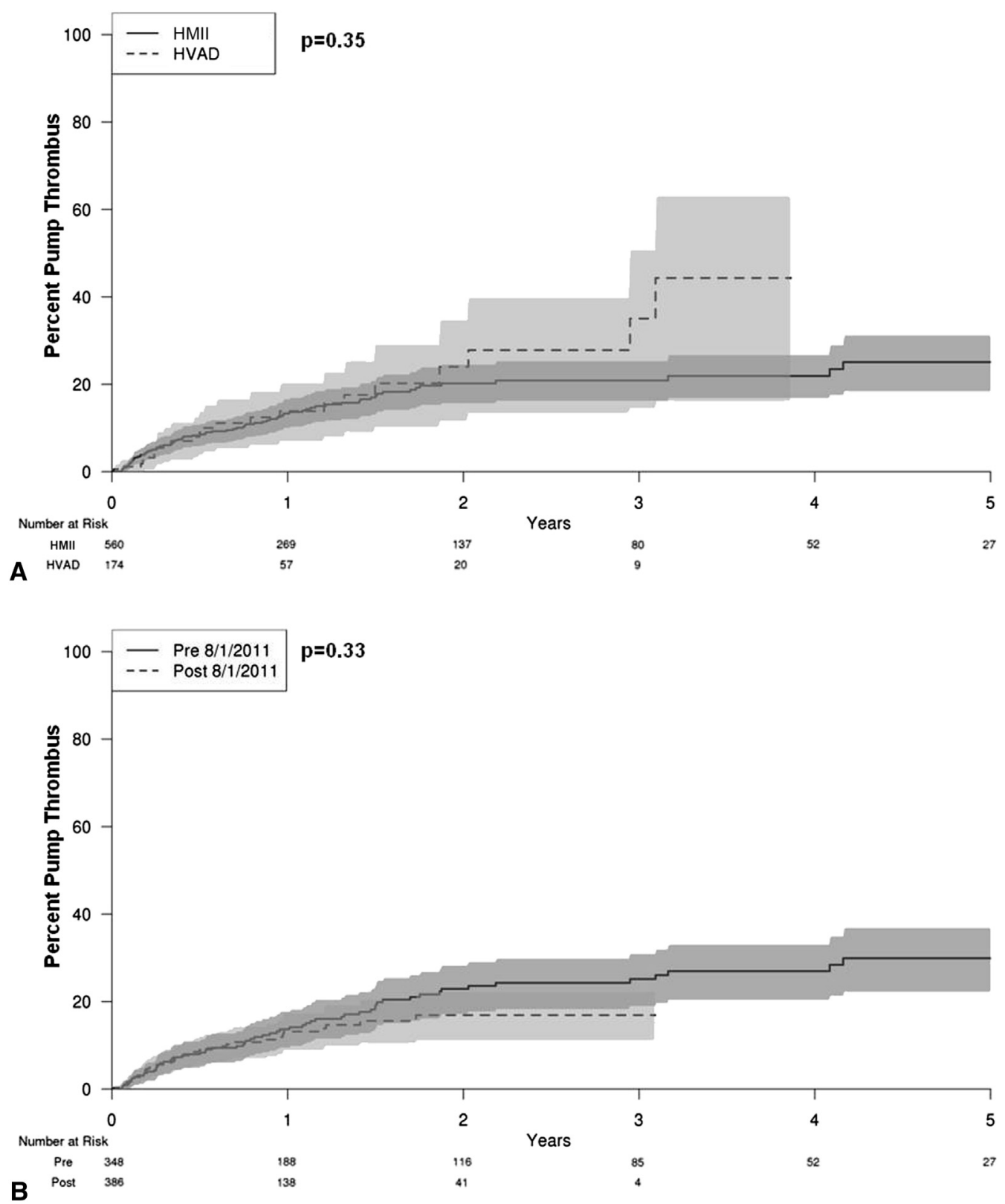

FIGURE 6. A, Time-related cumulative risk of pump thrombus for patients undergoing HMII (solid line) versus HVAD (dotted line) implantation; no difference was found, with data stratified by device type $(P=.35)$. B, No difference was found in cumulative risk of pump thrombus before (solid line), versus after, August 2011 (dotted line) $(P=.33)$. HMII, HeartMate II; HVAD, HeartWare ventricular assist device.

Multiple events during the follow-up were recorded for stroke, pump thrombus, and driveline infection, as well as the incidence of events per patient-year (Table 3). A comparison of the overall risk of adverse events with the HVAD versus the HMII is shown in Figure 7.

\section{DISCUSSION}

Our analysis detected significant preoperative and operative characteristics between patient cohorts, as well as disparities in late outcomes. We observed a higher time- related cumulative risk of any infection $(P=.039)$, and stroke $(P=.006)$, in patients supported with the HVAD. When overall incidence of adverse events was analyzed as events per patient-year, we observed a trend toward a higher incidence of stroke $(P=.05)$ and pump thrombus $(P=.09)$ in the HVAD group, whereas a trend toward a higher incidence of percutaneous driveline infection was found in the HMII group $(P=.051)$. When device type was forced into a multivariable analysis to determine if it had any independent association with adverse outcomes, the HVAD was found to 
TABLE 3. Adverse events per patient-year, stratified by device type

\begin{tabular}{lcccc}
\hline Adverse event & $\begin{array}{c}\text { HMII } \\
(\mathbf{n}=\mathbf{5 6 0})\end{array}$ & $\begin{array}{c}\text { HVAD } \\
(\mathbf{n}=\mathbf{1 7 4})\end{array}$ & $\begin{array}{c}\text { Total } \\
(\mathbf{n}=\mathbf{7 3 4})\end{array}$ & $\boldsymbol{P}$ value \\
\hline Stroke & 0.11 & 0.26 & 0.14 & .05 \\
TIA & 0.02 & 0.02 & 0.02 & .7 \\
Ischemic & 0.05 & 0.09 & 0.06 & .2 \\
Hemorrhagic & 0.03 & 0.08 & 0.04 & .007 \\
Pump thrombus & 0.1 & 0.2 & 0.12 & .09 \\
Driveline infection & 0.12 & 0.056 & 0.09 & .051 \\
\hline
\end{tabular}

HMII, HeartMate II; HVAD, HeartWare ventricular assist device; TIA, transient ischemic attack.

be independently associated with a higher risk of stroke (HR [95\% CI]: $1.8[1.25,2.5] ; P=.003$ ).

This report represents a large, multicenter, collaborative effort and evaluates outcomes with an "all-comers" strategy. The Mechanical Circulatory Support Research Network was formally established in 2013 and represents a voluntary effort of investigators from: the University of Michigan Health System (Ann Arbor, Mich); the Mayo Clinic College of Medicine (Rochester, Minn); Vanderbilt Heart \& Vascular Institute (Nashville, Tenn); St Vincent Heart Center (Indianapolis, Ind); and Inova Fairfax Hospital (Fairfax, Va). Data collection is supervised directly by the principal investigators, as are data entry and maintenance.

This large, collaborative effort contains some patients who were implanted as part of a clinical trial, as well as some implanted in a commercial setting. Very few efforts of this type have been undertaken; thus, we believe this to be a major strength of the current study, in that it offers a real-world experience and a diverse clinical collective from which to analyze a wide array of clinical outcomes with enhanced granularity. We believe that what distinguishes this effort from others is its more-detailed

\section{AE Risk Comparison}

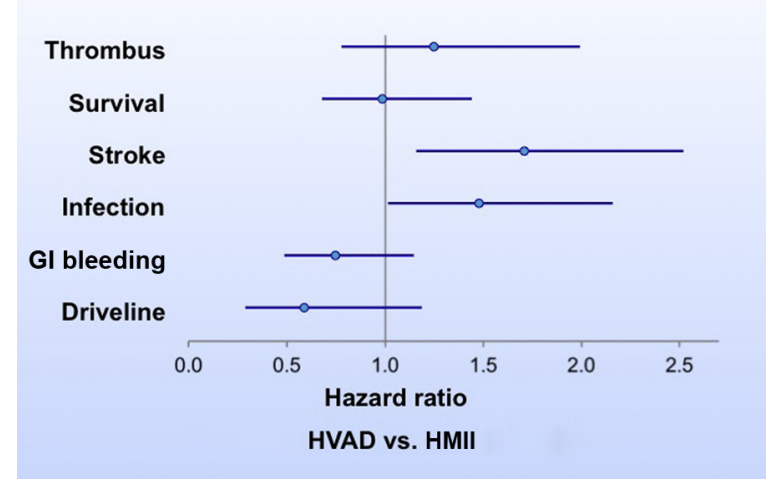

FIGURE 7. Adverse event risk comparison of the HVAD versus the HMII. The HVAD was associated with a higher risk of stroke and any infection, and a trend toward increased percutaneous driveline infection. AE, Adverse event; GI, gastrointestinal; HVAD, HeartWare ventricular assist device; HMII, HeartMate II. laboratory, echocardiographic, and hemodynamic assessments, as well as the robust readmissions data, including specifics on diagnosis and treatment of adverse events.

Our findings are similar to and confirm results of numerous reports, in many respects. Survival was not found to be significantly different with stratification by device. Longer cardiopulmonary bypass time, reflecting a more complex operation, and higher preoperative creatinine were found to be independently associated with lower late survival. The impact of concomitant procedures at the time of LVAD implantation has been reported to confer higher early mortality. ${ }^{6}$

Patients in our series who had the HMII more often required tricuspid valve surgery, but performance of this procedure alone was not shown to confer earlier risk or decreased late survival. More tricuspid valve procedures may reflect a larger destination therapy population in the HMII cohort, and although we did not stratify destination therapy and bridge to transplant specifically, both were entered into the multivariable model, and no differences in late survival were detected. The impact of renal dysfunction in patients undergoing transplantation is well documented and has been shown to portend inferior early and late outcomes. ${ }^{7}$

Gastrointestinal bleeding is a known complication after LVAD implantation and is multifactorial in nature. ${ }^{8,9}$ Our observations for postoperative GI bleeding are similar to large reports on both devices, ${ }^{10,11}$ and we did not detect any significant differences between patients' level of support on either device. Predictors of increased GI bleeding in this report reflect an older, sicker, and more-complex patient population, with older age at implant, redo sternotomy, higher preoperative creatinine, and preoperative inotrope use identified as being independently associated.

The risk of bleeding after HMII implantation was found to be highest when the INR was $>2.5$, and subsequent recommendations were made to maintain an INR goal of 1.5 to 2.5 to reduce this risk. ${ }^{10}$ This goal, of course, needs to be balanced with the potential higher risk of thromboembolic events, which are sometimes seen with lower INR levels. We did not stratify patients in this study according to INR levels during follow-up, but as we collect data prospectively, we will have this level of granularity in our followup data collection for these patients, so this area certainly is one to target in future investigations. Another issue that can benefit from further clarification in this dataset is the variation in protocols regarding anticoagulation and practice, according to device and era of implant.

Infection after LVAD implantation is well reported and has been shown to portend adverse outcomes and a significant increase in cost of care. ${ }^{12,13}$ A large report from the ADVANCE Bridge-to-Transplant and Continued Access Protocol trial ${ }^{14}$ documented lower percutaneous driveline infections and sepsis, compared with pivotal trials 


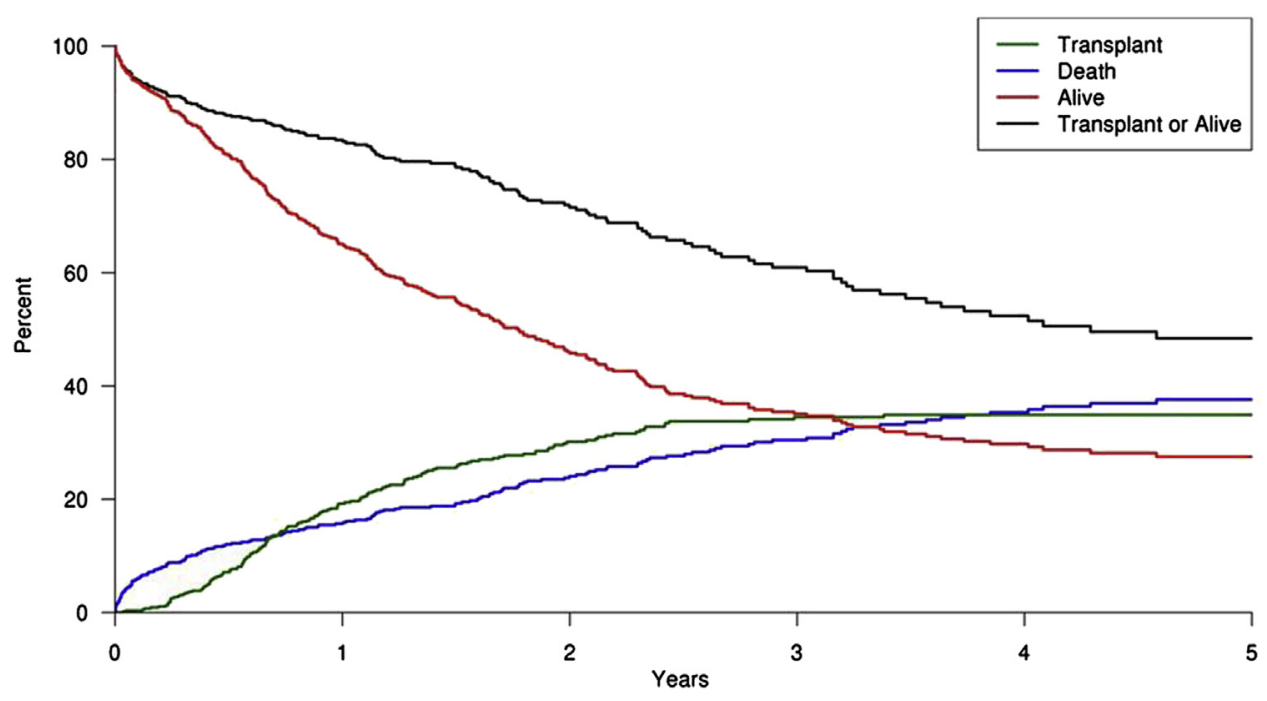

FIGURE 8. Competing outcomes are presented for patients after implantation with the HMII left ventricular assist device.

investigating the HMII, ${ }^{15,16}$ and our results demonstrate a lower incidence compared with both of these observations.

In analysis using stratification by device, we observed a significantly higher time-related cumulative risk of any infection, but no significant difference for driveline infection. An evaluation of driveline infection events per patient-year of support showed a trend toward a significantly higher incidence in HMII patients, compared with those who had an HVAD. Older age at implant was the common independent predictor of any infection, as well as driveline infection. Although this finding may reflect a higher proportion of destination therapy patients in the HMII group, this designation for LVAD was entered into the multivariable model as well, and found to not be an independent predictor. This result is a bit contrary to prior observations that longer duration of support significantly increases the risk of driveline infection, and hence, the greater risk is expected to occur in destination therapy patients. ${ }^{17}$ Although a more detailed analysis of the infection types the patients in this cohort sustained would be useful, we noted a greater incidence of pneumonia and bacteremia in the HVAD patients. This finding is surprising given the smaller pump size, lack of need for a pump pocket, and smaller caliber percutaneous driveline; all of these factors would be anticipated to facilitate implantation, lower pulmonary complications, and decrease device-related infections.

Perioperative antibiotic prophylaxis varied among centers included in this consortium and was not standardized. Furthermore, some centers advocate and practice chronic antibiotic administration for driveline infection prophylaxis. These differences provide an opportunity to investigate and analyze their relationship to efficacy and outcomes.

Neurologic complications are among the principal causes of morbidity and mortality after LVAD implantation. We observed a significantly higher time-related cumulative risk of stroke with the HVAD, and in a multivariable model, this risk was additionally identified as an independent predictor of stroke. These results are similar to those found in other comparisons of these 2 devices, which demonstrate a higher incidence of stroke with the HVAD. ${ }^{2-5}$ The absolute rate of stroke reported after continuous flow LVAD implantation has been noted to gradually decrease over time, with both the HMII ${ }^{1,15,18}$ and the HVAD, ${ }^{2,4,5}$ owing to practice changes and improved knowledge of the device and postoperative care.

We did not identify many predictors of stroke after LVAD implantation that other authors have, including age, ${ }^{10}$ female gender, ${ }^{10}$ diabetes mellitus,${ }^{19}$ hypertension, and atrial fibrillation. ${ }^{20}$ Rather, in addition to HVAD, ischemic etiology for heart failure was the only preoperative independent predictor for stroke in this series. This finding is not entirely surprising, given the known concomitant peripheral and vascular disease in patients with ischemic heart disease. Certainly, the cause of stroke after LVAD implantation is multifactorial, and relates to not only predisposing comorbidities, but also postimplantation patient management.

After implantation, patients possess both bleeding and clotting tendencies, as a result of chronic anticoagulation and biocompatibility with pump surfaces. We did not specifically analyze INR levels or blood pressure in this study. These parameters and their relationship to neurologic outcomes are critical to examine in future work.

A major design change to the HVAD was made in August 2011, which included sintering of the inflow cannula. We performed a deeper analysis of stroke in patients who were implanted with the HVAD before $(n=28)$ and after $(\mathrm{n}=146)$ this manufacturing change. The cumulative risk of stroke at 2 years was $36 \%$ before sintering, and $28 \%$ 


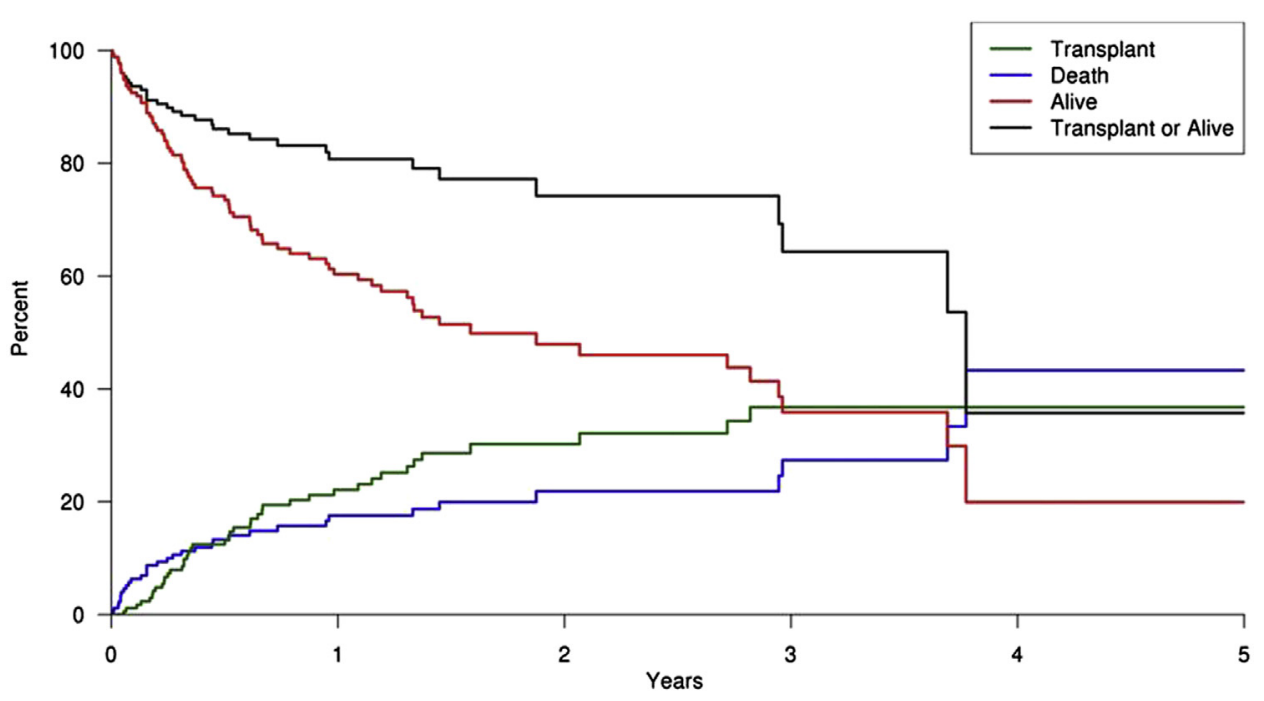

FIGURE 9. Competing outcomes are presented for patients after implantation with the HVAD.

after sintering $(P=.33)$. Although the risk was lower afterward, we believe the lack of a significant difference was mainly the result of the small number of subjects in the pre-sintering group. We acknowledge that stroke rates were lower in these patients, and that these may have been a result of this design change, along with application of greater vigilance to keeping blood pressure lower and of more-intense antiplatelet therapy. ${ }^{5}$

In both the clinical trial setting and the postapproval era, numerous series have documented an unavoidable rate of pump thrombosis with both the HMII and HVAD. ${ }^{18,21-24}$ Concern regarding HMII LVAD thrombosis has been heightened in the past year because of 2 publications. The first is an initial report ${ }^{21}$ by investigators who documented an abrupt increase in the incidence of confirmed pump thrombosis at 3 months, with the HMII-from $2.2 \%$ before March 2011 to $8.4 \%$ after January 2013. The second ${ }^{22}$ is a detailed analysis of the INTERMACS database, which reported a 6-fold increase in rates of pump thrombosis in the 2011 to 2012 timeframe. By contrast, the 6-month freedom, from device exchange and/or death because of pump thrombosis, fell significantly-from $99 \%$ in 2008 to 2009 , down to $95 \%$ in 2011 , and $94 \%$ in 2012 .

An important point to note for medical management is the temporal relationship of these findings to reports documenting the safe adoption of lower anticoagulation levels in patients who have the HMII, including the safe nonuse of perioperative intravenous heparin. ${ }^{9,25}$ Unfortunately, despite these data trends, firm conclusions cannot be drawn as to their causality, or whether they are patient- or device-specific. Additionally, the answers to many of these questions are being investigated by the ongoing Prevention of HeartMate II Pump Thrombosis Through Clinical Management (PREVENT) trial, in which pump thrombosis will be assessed when predefined practices for clinical management are adopted.
The HeartWare investigators, owing to concern about the high incidence of the HVAD pump thrombosis in their clinical trial, performed a detailed analysis ${ }^{23,26}$ and found that most pump thromboses occurred in patients who had subtherapeutic warfarin anticoagulation and were undergoing low-dose antiplatelet therapy or no antiplatelet therapy. After recommendations were made for strict patient management, to adhere to an INR of 2 to 3 , and administration of a high dose of aspirin $(325 \mathrm{mg})$, the rate of device exchange that was due to pump thrombosis decreased by $>50 \%$.

Because temporal analysis of pump thrombosis is the focus of an upcoming publication, we did not perform this in the current study. But in the entire cohort, no difference was found between devices in time-related cumulative risk of pump thrombosis, whereas a trend was found toward a higher incidence of events per patient-year in the HVAD group. A lower INTERMACS profile was the only independent predictor of pump thrombosis in this cohort, and neither device was independently associated with this outcome.

Furthermore, we did not observe any difference of cumulative risk of pump thrombus for patients implanted before versus after sintering. We attribute this finding in large part to the small number of HVAD patients implanted before sintering in this network ( 28 before, vs 146 after, sintering). However, an analysis of the true incidence rate showed a significant decrease in pump thrombus before and after 2011. In the current era, our general approach to surgical device exchange is to make a left subcostal incision for the HMII and to perform a repeat media sternotomy for the HVAD. Prior to approximately 2011, HMII device exchanges were performed via a repeat median sternotomy, using a curved left subcostal incision ("hockey stick"). This approach was used before it was fully appreciated that the pump housing could be approached and exchanged with very little dissection of the outflow graft or apex. Given 
that the HVAD is an intrapericardial pump, we continue to utilize the median sternotomy method.

\section{Limitations}

This study is a retrospective analysis of a patient registry shared among multiple institutions, and has the limitations inherent in this type of study. Despite robust patient cohort numbers, and multiregion sampling, this patient population may not be representative of LVAD patients at other institutions. This study includes data from 3 institutions, limited by missing data fields and potential entry inconsistencies. Although we believe the wide timespan of the study period is a strength of this effort, arguably heterogeneity is introduced via analysis of patients from both a clinical trial and a postapproval setting.

In addition, data were missing from our registry, and we employed pairwise deletion to impute these. Although this approach may have introduced an inherent level of bias, we believe the missing data occurred at random, thus minimizing any such bias; more likely, this imputation had a greater adverse effect on the power of the analysis. Collection and classification of adverse events were verified, and no events were self-reported. Although we believe such discrepancies are minimal, definitions of adverse events have evolved over time; similarly, our classification of these events changed along with changes made in INTERMACS classifications. Competing outcome curves for the HMII and HVAD are presented in Figures 8 and 9, respectively.

\section{CONCLUSIONS}

In this large, multicenter, collaborative analysis of adverse events among the contemporary 2 nd- and 3rd-generation continuous-flow LVADs, a trend (not statistically significant) was found toward a higher incidence of percutaneous driveline infections in patients treated with the HMII. A higher incidence of stroke was found in patients treated with the HVAD. Analysis of the true incidence of pump thrombus showed a significant decrease in pump thrombus for patients implanted before versus after 2011. Although the time-related cumulative risk of any infection and of stroke were significantly higher in the HVAD group, the HVAD was independently associated with stroke only. Understanding these differences can significantly enhance preoperative counseling, as well as postoperative monitoring of patients.

Large, multicenter collaborations are needed on an ongoing basis, to enhance our understanding of these critical issues and provide added appreciation of the deficiencies of this therapy. Collaborations that make use of these real-world multicenter efforts along with the large databases, such as INTERMACS and those from clinical trials, could propel the field toward establishment of guidelines and best practices, through comparison of clinical approaches and the resultant outcomes.

\section{Conflict of Interest Statement}

J.C., F.D.P., K.D.A., and S.M. report grant support from HeartWare Inc. S.M. reports grant support from ClearFlow. S.D., K.D.A., and F.D.P. report grant support from NHLBI. S.D. reports grant support from Patient-Centered Outcomes Research Institute. All other authors have nothing to disclose with regard to commercial support.

\section{References}

1. Kirklin JK, Naftel DC, Pagani F, Kormos RL, Stevenson LW, Blume ED, et al. Sixth INTERMACS annual report: a 10,000-patient database. J Heart Lung Transplant. 2014;33:555-64.

2. Aaronson KD, Slaughter MS, Miller LW, McGee EC, Cotts WG, Acker MA, et al. Use of an intrapericardial, continuous-flow, centrifugal pump in patients awaiting heart transplantation. Circulation. 2012;125:3191-200.

3. Lalonde SD, Alba AC, Rigobon A, Ross HJ, Delgado DH, Billia F, et al. Clinical differences between continuous flow ventricular assist devices: A comparison between HeartMate II and HeartWare HVAD. J Card Surg. 2013; 28:604-10.

4. US Food and Drug Administration. Summary of safety and effectiveness data (SSED). HeartWare Ventricular Assist System P100047. Available at: http://www. accessdata.fda.gove/scripts/cdrh/cfdocs/cftopic/pma/pma.cfm?num=p100047. Accessed August 23, 2015.

5. Pagani FD, Milano CA, Tatooles AJ, Bhat MS, Slaughter EJ, Birks EJ, et al. HeartWare HVAD for the treatment of patients with advanced heart failure ineligible for cardiac transplantation: results of the ENDURANCE Destination Therapy trial. J Heart Lung Transplant. 2015;34:S9 [Abstract].

6. John R, Naka Y, Park SJ, Sai-Sudhakar C, Salerno C, Sundareswaran KS, et al. Impact of concurrent surgical valve procedures in patients receiving continuousflow devices. J Thorac Cardiovasc Surg. 2014;147:581-9.

7. Coffin ST, Waguespack DR, Haglund NA, Maltais S, Dwyer JP, Keebler ME. Kidney dysfunction and left ventricular assist device support: a comprehensive perioperative review. Cardiorenal Med. 2015;5:48-60.

8. Crow S, Chen D, Milano C, Thomas W, Joyce L, Piacentino V III, et al. Acquired von Willebrand syndrome in continuous-flow ventricular assist device recipients. Ann Thorac Surg. 2010;90:1263-9; discussion 1269.

9. Boyle AJ, Russell SD, Teuteberg JJ, Slaughter MS, Moazami N, Pagani FD, et al. Low thromboembolism and pump thrombosis with the HeartMate II left ventricular assist device: analysis of outpatient anticoagulation. J Heart Lung Transplant. 2009;28:881-7.

10. Boyle AJ, Jorde UP, Sun B, Park SJ, Milano CA, Frazier OH, et al. Preoperative risk factors of bleeding and stroke during left ventricular assist device support: an analysis of more than 900 HeartMate II outpatients. J Am Coll Cardiol. 2014;63: $880-8$.

11. Maltais S, Ewald GA, Keebler ME, Boyce SS, Najjar FD, Pagani FD, et al. Update on post-approval INTERMACS registry of the HVAD system in commercial use. J Heart Lung Transplant. 2015;34:S195 [Abstract].

12. Goldstein DJ, Naftel D, Holman W, Bellumkonda L, Pamboukian SV, Pagani FD, et al. Continuous-flow devices and percutaneous site infections: clinical outcomes. J Heart Lung Transplant. 2012;31:1151-7.

13. Slaughter MS, Bostic R, Tong K, Russo M, Rogers JG. Temporal changes in hospital costs for left ventricular assist device implantation. J Card Surg. 2011;26: 535-41.

14. John R, Aaronson KD, Pae WE, Acker MA, Hathaway DR, Najarian KB, et al; HeartWare Bridge to Transplant ADVANCE Trial Investigators. Driveline infections and sepsis in patients receiving the HVAD system as a left ventricular assist device. J Heart Lung Transplant. 2014;33:1066-73.

15. Miller LW, Pagani FD, Russell SD, John R, Boyle AJ, Aaronson KD, et al; Heart Mate II Clinical Investigators. Use of a continuous-flow device in patients await ing heart transplantation. N Engl J Med. 2007;357:885-96.

16. Pagani FD, Miller LW, Russell SD, Aaronson KD, John R, Boyle AJ, et al Extended mechanical circulatory support with a continuous-flow rotary left ventricular assist device. J Am Coll Cardiol. 2009;54:312-21.

17. Sharma V, Deo SV, Stulak JM, Durham LA III, Daly RC, Park SJ, et al. Driveline infections in left ventricular assist devices: implications for destination therapy Ann Thorac Surg. 2012;94:1381-6.

18. Park SJ, Milano CA, Tatooles AJ, Rogers JG, Adamson RM, Steidley DE, et al; HeartMate II Clinical Investigators. Outcomes in advanced heart failure patients 
with left ventricular assist devices for destination therapy. Circ Heart Fail. 2012; $5: 241-8$.

19. Morgan JA, Brewer RJ, Nemeh HW, Gerlach B, Lanfear DE, Williams CT, et al. Stroke while on long-term left ventricular assist device support: incidence, outcome, and predictors. ASAIO J. 2014;60:284-9.

20. Stulak JM, Deo S, Schirger J, Aaronson KD, Park SJ, Joyce LD, et al. Preoperative atrial fibrillation increases risk of thromboembolic events after left ventricular assist device implantation. Ann Thorac Surg. 2013;96:2161-7.

21. Starling RC, Moazami N, Silvestry SC, Ewald G, Rogers JG, Milano CA, et al. Unexpected abrupt increase in left ventricular assist device thrombosis. $N$ Engl J Med. 2014;370:33-40.

22. Kirklin JK, Naftel DC, Kormos RL, Pagani FD, Myers SL, Stevenson LW, et al. Interagency Registry for Mechanically Assisted Circulatory Support (INTERMACS) analysis of pump thrombosis in the HeartMate II left ventricular assist device. J Heart Lung Transplant. 2014;33:12-22.

23. Najjar SS, Slaughter MS, Pagani FD, Starling RC, McGee EC, Eckman P, et al. An analysis of pump thrombus events in patients in the HeartWare ADVANCE bridge to transplant and continued access protocol trial. J Heart Lung Transplant. 2014;33:23-34.

24. Stulak JM, Maltais S. A different perspective on thrombosis and the HeartMate II. N Engl J Med. 2014;370:1467-8.

25. Slaughter MS, Naka Y, John R, Boyle A, Conte JV, Russell SD, et al. Postoperative heparin may not be required for transitioning patients with a HeartMate II left ventricular assist system to long-term warfarin therapy. J Heart Lung Transplant. 2010;29:616-24.

26. Slaughter MS, Pagani FD, McGee EC, Birks EJ, Cotts WG, Gregoric I, et al. HeartWare ventricular assist system for bridge to transplant: combined results of the bridge to transplant and continued access protocol trial. J Heart Lung Transplant. 2013;32:675-83.

Key Words: mechanical circulatory support, heart failure, ventricular assist device

\section{Discussion}

Dr J. Kirklin (Birmingham, Al). I would like to congratulate the authors for a very nice multi-institutional analysis, and I have 2 very concrete questions for you. It is apparent now that the HVAD has been associated with an increasing incidence of neurologic events in several studies. So, first, what do you hypothesize is, potentially, the difference between axial-flow and centrifugal-flow pumps in this regard?

Dr J. Stulak. Thank you, Dr Kirklin. It is an honor for you to be our discussant. The differences in observed adverse neurologic outcomes between these 2 pump technologies have been consistent, and I believe, represent both pump- and patient-related factors. The HVAD is more sensitive to afterload than the HMII, and as we know, these patients require a full aspirin, rather than the typical baby aspirin required for HMII patients. Appreciation of these differences has improved outcomes, but there is certainly more attention paid to the inflow cannula of the HVAD and whether the sintering that was performed was adequate. Personally, I still see a large amount of pannus in the left ventricular cavity during HVAD device exchange. So, this is also a contributing factor.

Dr Kirklin. Also, the driveline infections are interesting. Even though there is some variability in your statistical analysis, it looks like there is a continuous hazard for driveline infections in the HMII. To what do you attribute that?

Dr Stulak. Certainly the drivelines are different as well. I am uncertain if the major issue is a larger caliber driveline, or if the difference reflects a longer time on support for the HMII patients. The curves for infection diverge at approximately 1 year; perhaps the longer a patient is on support, the greater the continuous hazard for having a driveline infection. The other possibility is that an older, sicker, destination-therapy population is reflected more in the HMII patient population. This was an all-comers' strategy, and we did not stratify by bridge-to-transplant and destination therapy, and I wonder whether, if we had, we might have seen that the destination therapy patients are older and have other comorbidities.

Dr M. Acker (Philadelphia, $\mathrm{Pa}$ ). Have you changed your blood pressure control strategy, as suggested by the HVAD ENDURANCE recommendations, and have you seen any difference in their stroke rate over the last year perhaps?

Dr Stulak. Yes-both anticoagulation and blood pressure management have changed. Having said that, we have had several HVAD device exchanges recently, so I am not personally convinced that the incidence is decreasing after these measures, at least from my experience.

Dr Acker. In stroke?

Dr Stulak. In stroke and pump thrombus, unfortunately.

Dr H. Reichenspurner (Hamburg, Germany). There is, however, a striking difference in the stroke rate in the American trial compared to the European trial, such as the ReVOLVE (Registry to Evaluate the HeartWare Left Ventricular Assist System) trial. We discussed this at the recent ISHLT (International Society for Heart and Lung Transplantation) meeting extensively, and we really thought that blood pressure management and anticoagulation management might make a big difference. So I think it is important to emphasize that in the ReVOLVE trial, there was actually a much lower incidence among the European centers using the HVAD.

Dr Stulak. I believe this is why more multi-institutional studies are needed, even international collaborations, in order to analyze the practice variations, the differences in patient populations, and finally, how we are practicing differently.

Dr A. Anyanwu (New York, NY). When you counsel your patients who are getting an HVAD, do you tell them there is a higher stroke risk now or not?

Dr Stulak. We tell them that there are pros and cons to both the device-

Dr Anyanwu. But do you tell them there is a higher stroke rate, yes or no?

Dr Stulak. Yes, we do. We have to. These are the data in every study to date. 